\title{
Clinical outcomes in patients with lung cancer admitted to intensive care units
}

\author{
Jinkyeong Park ${ }^{1}$, Woo Jin Kim², Ji Young Hong ${ }^{3}$, Yoonki Hong ${ }^{2}$ \\ ${ }^{1}$ Department of Internal Medicine, Dongguk University Ilsan Hospital, Goyang, Republic of Korea; ${ }^{2}$ Department of Internal Medicine, School \\ of Medicine, Kangwon National University, Kangwon National University Hospital, Chuncheon, Republic of Korea; ${ }^{3}$ Division of Pulmonary and \\ Critical Care Medicine, Department of Medicine, Chuncheon Sacred Heart Hospital, Hallym University Medical Center, Chuncheon, Republic of \\ Korea \\ Contributions: (I) Conception and design: J Park, Y Hong; (II) Administrative support: All authors; (III) Provision of study materials or patients: J \\ Park, JY Hong, Y Hong; (IV) Collection and assembly of data: J Park, Y Hong; (V) Data analysis and interpretation: J Park, JY Hong, Y Hong; \\ (VI) Manuscript writing: All authors; (VII) Final approval of manuscript: All authors. \\ Correspondence to: Yoonki Hong, MD. Department of Internal Medicine, School of Medicine, Kangwon National University, 1 Gangwondaehak-gil, \\ Chuncheon 24341, Republic of Korea. Email: h-doctor@hanmail.net.
}

Background: Recent advances in critical care and infection control have led to improved intensive care unit (ICU) survival rates. However, controversy exists regarding the benefits of ICU treatment for patients with lung cancer. In this study, we evaluated the clinical outcomes of patients from the Korean national database, who had been diagnosed with lung cancer and had received ICU treatment.

Methods: We investigated patients in Korea who had been newly diagnosed with lung cancer between January 1, 2008 and December 31, 2010. We classified these critically ill patients with lung cancer according to their lung cancer treatment pathways, with a specific focus on those who had undergone ICU treatment.

Results: We found that $31.3 \%$ of patients newly diagnosed with lung cancer had been admitted to the ICU for any reason, and $18.5 \%$ of patients with lung cancer were admitted to the ICU for reasons other than postoperative surgical lung cancer resection. The ICU mortality rate was $2.9 \%$ in patients admitted to the ICU for postoperative care and $47.5 \%$ in patients admitted for other reasons. Clinical cancer staging (HR, 7.02; 95\% CI, 5.82-8.48; $\mathrm{P}<0.01$ ) and the need for mechanical ventilator (HR, 1.34; 95\% CI, 1.27-1.41; $\mathrm{P}<0.01)$ were independently associated with ICU mortality. The importance of mechanical ventilator intervention as a predictor for survival was significantly greater in the earlier stages of lung cancer (HR, 1.97; 95\% CI, 1.15-3.38; $\mathrm{P}<0.01)$.

Conclusions: This study suggests that goals and treatment plans for critically ill patients with lung cancer should be determined by the individual patient's clinical cancer stage, regardless of the reason for admission to the ICU.

Keywords: Lung cancer; intensive care unit (ICU); mortality, survival rates

Submitted Jan 20, 2021. Accepted for publication Mar 21, 2021.

doi: $10.21037 / \mathrm{atm}-21-298$

View this article at: http://dx.doi.org/10.21037/atm-21-298

\section{Introduction}

Lung cancer is the leading cause of cancer death, accounting for $20 \%$ of all cancer deaths worldwide (1). Patients with lung cancer comprise $27 \%$ of all intensive care unit (ICU) admissions among patients with solid cancers $(2,3)$. A retrospective study reported that critically ill patients with lung cancer showed high mortality rates of up to $74 \%$ (4). Poor quality of life have also been reported in patients after discharge from the ICU (5).

Over the last two decades, significant advances in critical care and infection control have led to improved survival rates $(6,7)$. Recent studies indicate that further improvements 
in the survival rates of lung cancer patients treated in ICU may be expected $(5,8)$. Moreover, the introduction of new treatments, such as targeting agents or immunotherapy have also contributed to improved survival in patients with advanced lung cancer $(9,10)$. As a result of the complex nature of the disease and the growing number of treatment options for these patients, it has become difficult to apply past treatment decision recommendations (11) to the modern critical care of patients with lung cancer. To complicate matters further, in previous studies, the criteria for treatment-limitation decisions were different for each center, and the severity of patients admitted into ICU was influenced by the availability of beds in ICU at each hospital (12). Most studies have reported outcomes for all lung cancer patients, including surgical patients, whereas a few have focused only on advanced cancer patients who do not have surgical treatment options. There is therefore a need for unbiased data on outcomes for all-stage critically ill patients with lung cancer in ICUs in order to assist physicians and patients in making more informed and considered decisions regarding ICU treatment.

We hypothesized that the overall prognosis of patients with lung cancer in ICU was influenced by the cancer treatment the patient actually received, rather than by the cancer stage at the time of diagnosis. We aimed to investigate the outcomes of each clinical group by classifying critically ill patients with lung cancer from a national population-based data according to their lung cancer treatment options. We present the following article in accordance with the STROBE reporting checklist (available at http://dx.doi.org/10.21037/atm-21-298).

\section{Methods}

\section{Design and setting}

This is a retrospective observational cohort study. This study analyzed the claims data from the Health Insurance Review and Assessment Service (HIRA) between January 1, 2007 and March 31, 2016. All Koreans are covered by the National Health Insurance (NHI) and claims data submitted by the NHI are reviewed by the HIRA. These claims data include information regarding patients' diagnoses, treatment, procedures, surgical history, and prescription drugs.

\section{Patients}

This study investigated all patients aged $>18$ years, examined by chest CT for any reason between January 2007 to December 2011. From this cohort, we identified those patients with lung cancer using codes from their ICU services [i.e., code C34x.x from a modified version of the International Classification of Diseases 10th Revision (ICD-10)]. The study enrolled patients newly diagnosed lung cancer between 2008 and 2011 and who were followed up before March 31, 2016. We excluded patients who: (I) were $>100$ years old, (II) had any claims related to code C34x.x prior to Jan 2008, (III) had a lung cancer diagnosis after the date of admission to the ICU, (IV) stayed in the ICU less than $24 \mathrm{~h}$.

This left a cohort of 74,754 patients who were categorized into five clinical stages according to the treatment they received: (I) lung cancer resection only, (II) combined systemic therapy with lung cancer resection, (III) systemic therapy without lung cancer resection, (IV) no treatment, and $(\mathrm{V})$ refusal of any treatment. Among patients with no treatment, those without metastases were placed in the refusal group.

\section{Comorbidities and concomitant medical therapy}

We identified the first ICU admission after lung cancer diagnosis for each patient using codes from the ICU services (AJ001-AJ590900). Patients who stayed in ICU for $>24 \mathrm{~h}$ and those admitted for routine postoperative care were classified as surgical ICU. Radiation therapy for lung cancer was limited to radiation therapy administered to the chest area. Comorbidities were identified if the claims data existed from six months before the index diagnosed as lung cancer. Comorbidity diagnoses were identified using ICD-10 codes and where patients had underlying disease, comorbidity was represented by the Charlson Comorbidity index. Procedures of interest such as mechanical ventilation, hemodialysis, and cardiopulmonary resuscitation (CPR) were defined using procedural claim codes as detailed in a previous study (13). We identified the use of chemotoxic drugs using the Korean drug and anatomical therapeutic chemical codes.

\section{Ethics}

The study was conducted in accordance with the Declaration of Helsinki (as revised in 2013). The study was approved Kangwon National University Hospital Institutional Review Board (B-2018-02-002) and individual consent for this retrospective analysis was waived. 


\section{Statistical analysis}

All statistical analyses were performed with reference to guidelines for the European fournal of Cardio-Thoracic Surgery and the Interactive CardioVascular and Thoracic Surgery (14). The study endpoint was defined as allcause mortality in the ICU. Variables are presented as numbers (percentages) or as (means \pm standard deviations). Between-group comparisons were drawn using $\chi^{2}$ tests for categorical data and Student's $t$-tests for continuous data. We used Cox proportional regression to calculate the hazard ratios (HR) and $95 \%$ confidence intervals (95\% CI) for ICU treatments associated with patient characteristics. In order to avoid overcorrection of cancer disease, the value obtained by subtracting the cancer related score from Charlson Comorbidity Index was used. Probabilities of $\mathrm{P}<0.05$ were considered statistically significant. All analyses were carried out using $\mathrm{R}$ v3.4.4. We used the survival package and ggplot2 functions in $\mathrm{R}$ to get appropriate updated citations.

\section{Results}

Between 2008 and 2011, 74,754 patients were diagnosed with lung cancer. The follow-up duration was $926.7 \pm$ 961.8 days. Among patients with lung cancer, 23,365 (31.3\%) patients were admitted to the ICU. The mean period between cancer diagnosis and ICU admission was $242.9 \pm 461.9$ days. Patients treated with a wide variety of therapies comprised the greatest proportion (30.5\%) of all patients with lung cancer admitted to the ICU. Most patients admitted to the ICU (39.8\%) received systemic therapy without lung cancer resection; the second largest group admitted to the ICU $(27.7 \%)$ comprised patients who refused treatment for cancer. As lung cancer was newly diagnosed, the proportion of cancer-treated patients increased during the follow-up period (from $57.3 \%$ in 2008 to $61.5 \%$ in 2010 , Table 1). On average, patients with lung cancer followed for $1,131.3 \pm 994.1$ days and were treated in the ICU for $34.2 \pm 143.9$ days. Among patients admitted to the ICU, organ failure mainly occurred in the respiratory system (39.2\%) and renal system (4.6\%). The mortality rate of lung cancer patients admitted to the ICU was $24.4 \%$ at day $28,33.8 \%$ at day $60,49.9 \%$ at 1 year, and $61.4 \%$ at 3 -year post admission to the ICU. Mortality rate was related to clinical staging according to cancer treatment (Figure 1).

\section{Surgical versus medical ICU admission}

Characterization by type of admission to the ICU in patients with lung cancer is summarized in Table 2. ICU admission for postoperative care (surgical ICU) accounted for $40.6 \%$ of patients admitted to ICU. More patients who received chemotherapy and lung resection (58.4\%) were admitted into the ICU for postoperative care than those who received lung resection only. Patients with lung cancer stayed in medical ICU longer than in surgical ICU $(40.4 \pm 143.9$ vs. $25.1 \pm 123.3$ days, $\mathrm{P}<0.001)$. Surgical ICU showed significantly lower mortality rates compared to medical ICU at day $28(1.5 \%$ vs. $40.0 \%, \mathrm{P}<0.001)$ and day 60 (3.2\% vs. $54.7 \%, \mathrm{P}<0.001)$. CPR was performed in $93.3 \%$ of critically ill patients who died in the surgical ICU compared $86.3 \%$ of patients in medical ICU $(\mathrm{P}<0.001)$. Critically ill patients survived longer after discharge from surgical ICU than from medical ICU $(908.7 \pm 676.2 v s$. $127.5 \pm 296.9$ days, $\mathrm{P}<0.001)$.

\section{Clinical outcomes of ICU admission for nonsurgical reasons}

Characteristics of patients admitted to the ICU for nonsurgical reasons are summarized in Table 3. Patients treated with lung cancer resection alone received more renal replacement therapy $(12.8 \%$ vs. $5.8 \%, \mathrm{P}<0.001)$ and mechanical ventilation $(58.2 \%$ vs. $53.1 \%, \mathrm{P}<0.001)$ than those who were treated with systemic therapy alone. Among patients who received lung cancer resection, there was no difference in the use of ventilators between the patients who underwent surgery alone and those who received lung resection and systemic therapy together $(58.4 \% v s$. $58.2 \%, \mathrm{P}=0.11)$. However, the mortality rate at any point was remarkably higher among patients who received lung resection and systemic therapy (Figure 2). Patients who received lung cancer resection, regardless of whether or not this was combined with systemic cancer treatment, stayed in ICU longer than those who did not undergo resection and survived longer after discharge from ICU than non-resected patients. The prognostic effect of ventilators on outcomes in medical ICU was HR 1.97 (95\% CI, 1.15-3.38, P<0.01) in the group who received with only surgery, HR 1.85 (95\% CI, 1.37-2.52, $\mathrm{P}<0.01)$ in the group who received combined systemic therapy with surgery, HR 1.40 (95\% CI, 1.30-1.51, $\mathrm{P}<0.01)$ in the group who received systemic therapy without surgery, and HR 1.12 (95\% CI, 0.99-1.27, $\mathrm{P}=0.07$ ) in the 


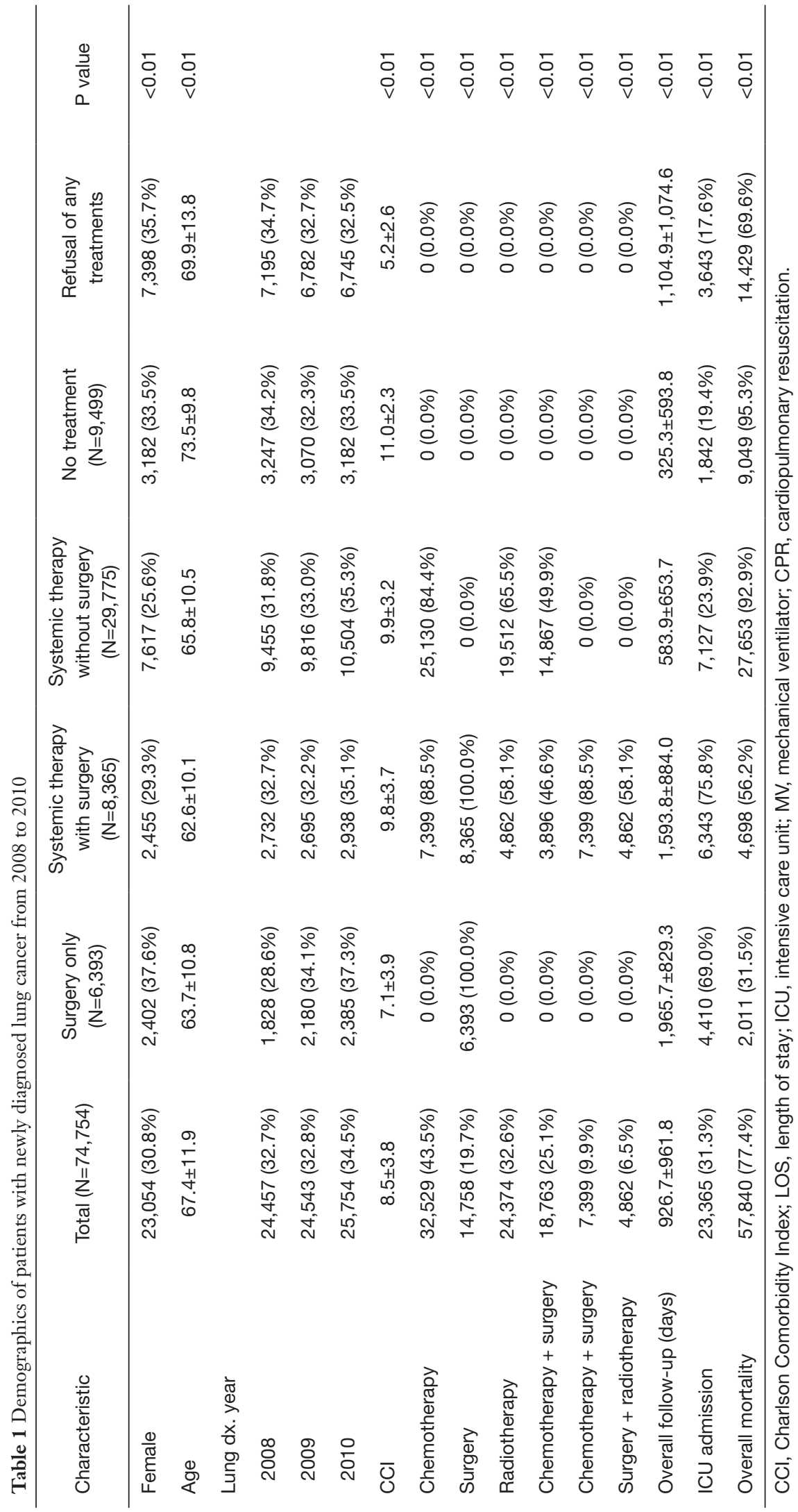




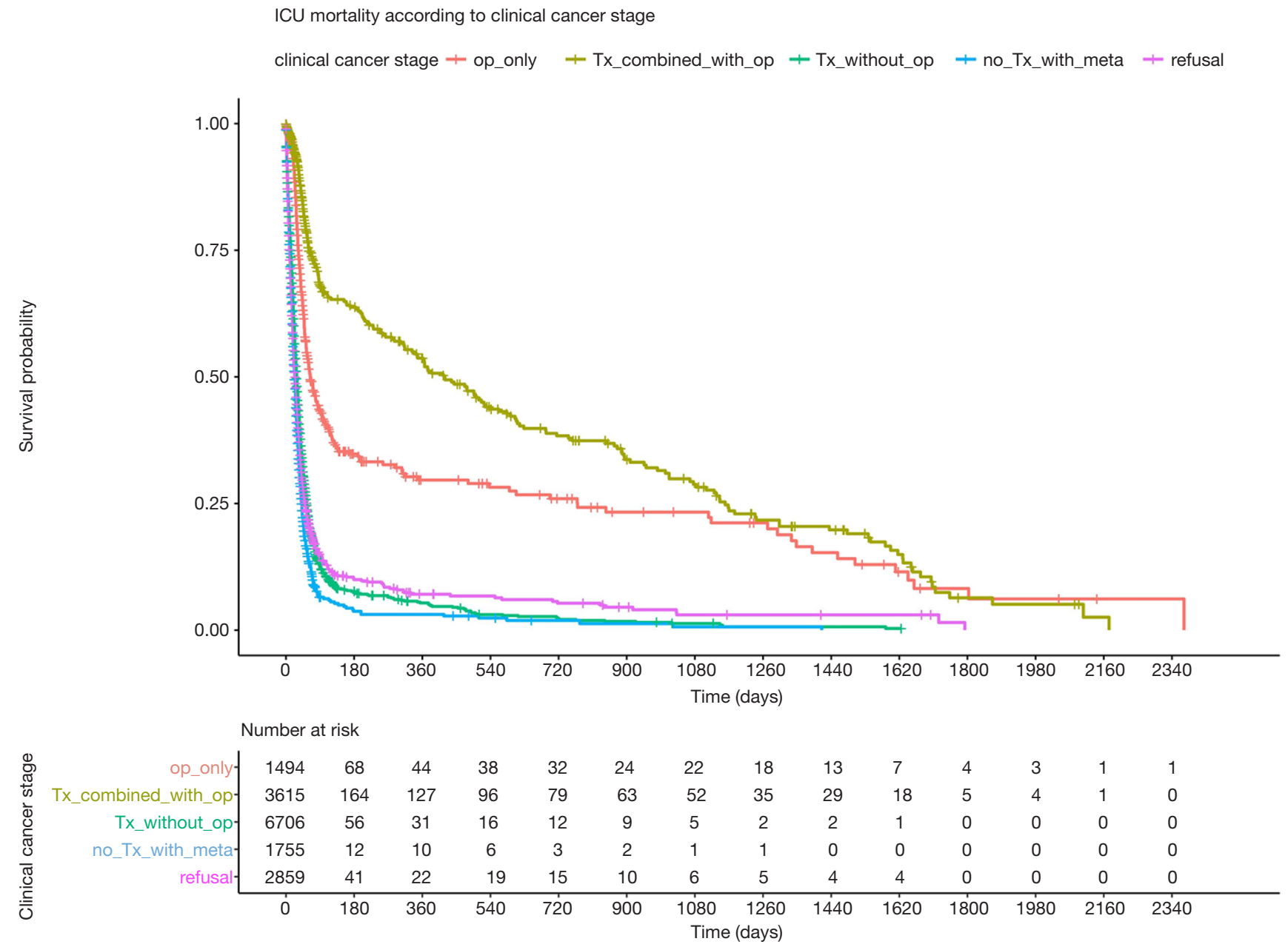

Figure 1 Intensive care unit mortality in lung cancer according to clinical stage. Op_only, lung cancer resection only; Tx_combined_with_ op, combined systemic therapy with lung cancer resection; Tx_without_op, systemic therapy without lung cancer resection; no_Tx_with_ meta, no treatment; refusal, refusal of any treatment.

group who did not receive any cancer-related treatment.

\section{Discussion}

This study shows the comprehensive outcomes of lung cancer patients admitting to ICU according to modality receiving treatment, based on a national population study. Treatment outcomes varied significantly depending on the clinical stage. Although there was no difference in the frequency of treatment with ventilators, patients with advanced lung cancer who did not receive treatment from mechanical ventilators showed a ten-fold higher risk of death than those with early lung cancer under the same treatment conditions. Our results suggest that while critical care has improved significantly, the biggest challenge faced by critically ill patients with lung cancer lies in the cancer stage itself.

In our study, $31.3 \%$ of all lung cancer patients were admitted to ICU, and $47.5 \%$ of those admitted, died in the ICU. The mortality rate of patients admitted to ICU after lung cancer resection was $2.9 \%$. The ICU mortality rate for patients with early-stage lung cancer admitted to ICU for reasons other than postoperative surgical lung cancer resection was $27.7 \%$. This is similar to mortality rates for early stage lung cancer reported in previous studies $(15,16)$. Reichner et al. (15) found that one in four $(25 \%)$ patients with NSCLC stage I died and one of two (50\%) patients with limited stage SCLC died in medical ICU. 
Table 2 Characteristics according to type of admission to the ICU in patients with lung cancer

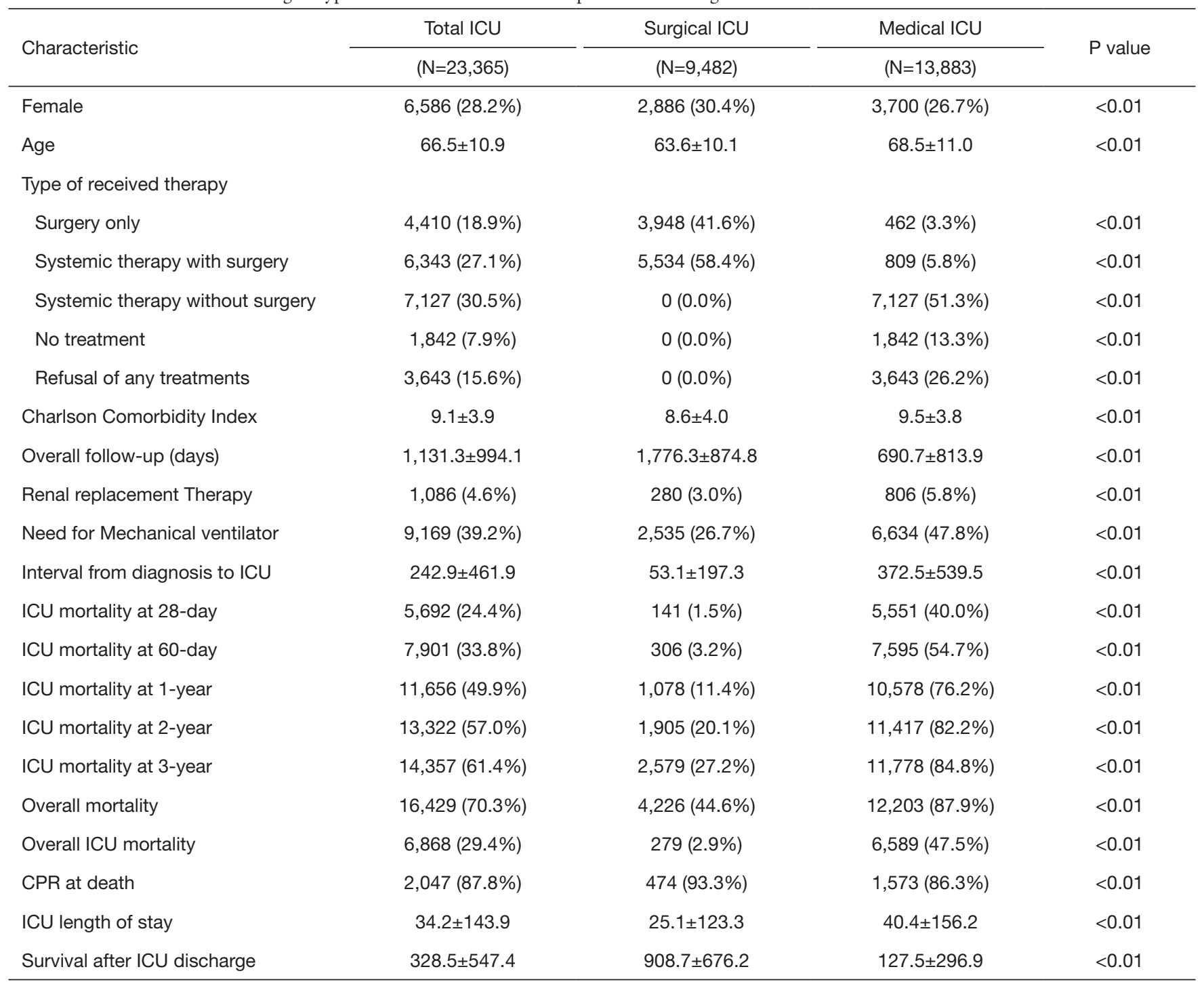

CPR, cardiopulmonary resuscitation.

Adam and Soubani (16) reported a 25\% ICU mortality rate among four patients with stage I NSCLC. However, too few patients with early stage cancer were included in these studies for meaningful conclusions to be drawn.

The decision to admit patients with advanced lung cancer to medical ICU continues to present a major challenge faced by doctors. Our results appear to suggest that the outcomes of medical ICU treatment in patients with resectable lung cancer are similar to those of critically ill patients having no cancer. Our study found an in-hospital mortality rate of $58.0 \%$, which was similar to those reported in most previous studies where medical ICU mortality rates of over $50 \%$ in patients with extensive or advanced lung cancer were reported. Our results also showed that $26.2 \%$ of patients with metastatic lung cancer were admitted to the ICU, despite being unable to receive cancer treatment. The mortality of these patients was $58.3 \%$. This was lower than the $68 \%$ mortality rate reported in the study by Reichner et al. (15) where stage IV patients made up $53.2 \%$ of all study patients. A study by Adam and Soubani (16) reported a significantly lower medical ICU mortality rate $(21.4 \%)$ in stage IV patients. However, their stage IV patients were on average ten years younger than those with metastatic cancer in our study. We noted a significant difference between 
Table 3 Characteristics of patients with lung cancer admitted to the ICU for nonsurgical reasons

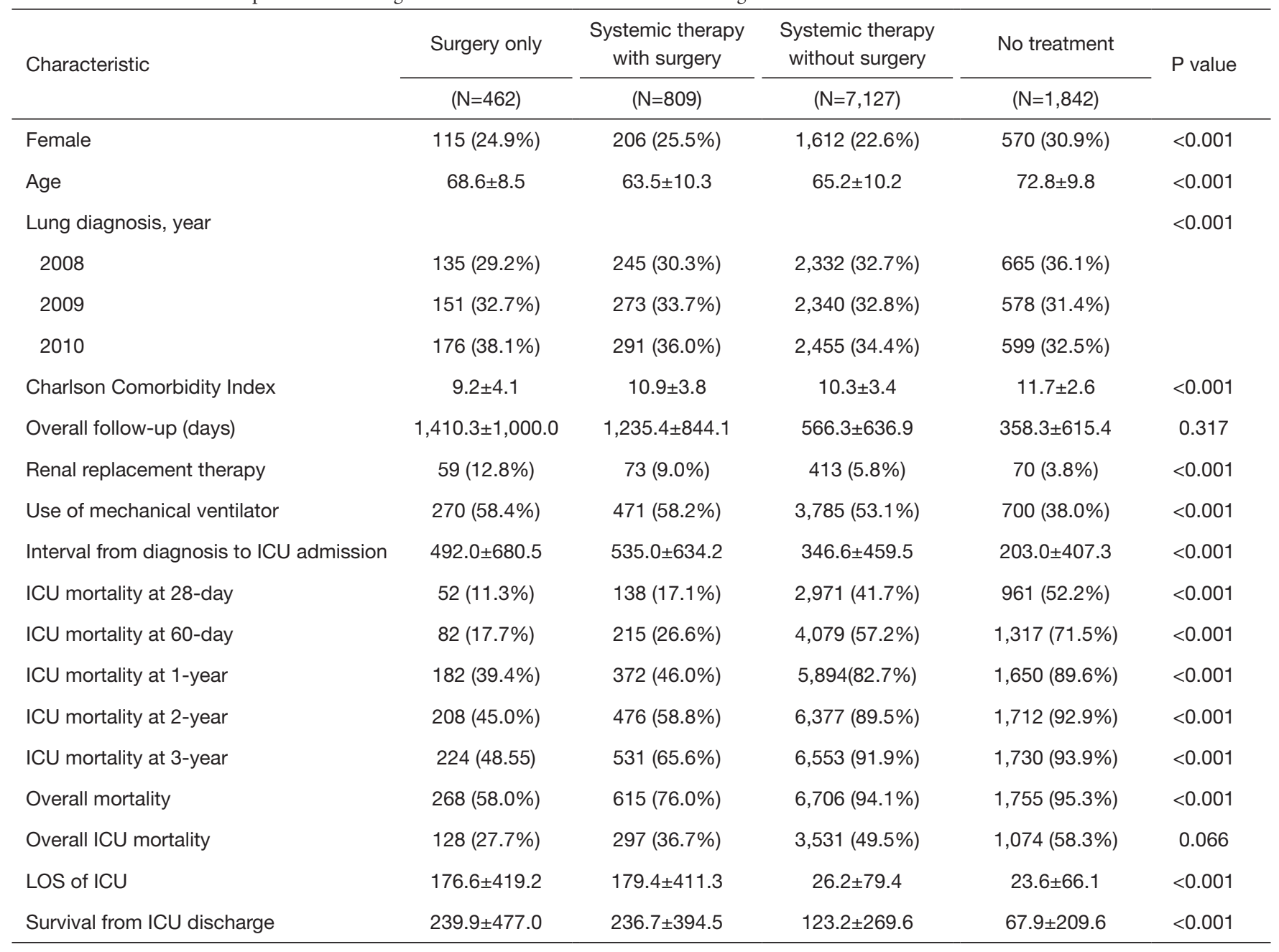

LOS, length of stay; ICU, intensive care unit.

outcomes of patients who did not receive any cancer-related treatment and those of patients with pathological stage IV. This suggests that whether cancer is controlled by cancerrelated treatments or not may be an important factor in medical ICU outcomes.

Previous studies $(4,8,17,18)$ reported that important predictors of ICU mortality included the need for mechanical ventilators, number of organ failures, performance status, and cancer recurrence or progression. In our multivariate Cox regression model, the stage of lung cancer and the need for a mechanical ventilator were correlated with ICU outcomes. The need for a mechanical ventilator was found to be an important factor for survival in patients with early lung cancer, whereas for patients with advanced lung cancer, the effect of the cancer itself was of greater importance than the need for mechanical ventilators. Advancements in the use of mechanical ventilators in critical care medicine might have led to improved survival in early lung cancer patients. However, the outcomes of medical ICU for advanced lung cancer patients remains poor, regardless of these developments.

Better understanding of clinical outcomes for lung cancer patients admitting ICU can be achieved from large sample sized or population based studies. A population based observational study in the Scotland region showed that lung cancer were most common cases of ICU admission for emergency medical reasons and had greatest mortality of $60 \%$ among solid cancers, except lowest mortality in cases of postoperative ICU care (19). Another cohort study reported trends and risk factors of mortality 


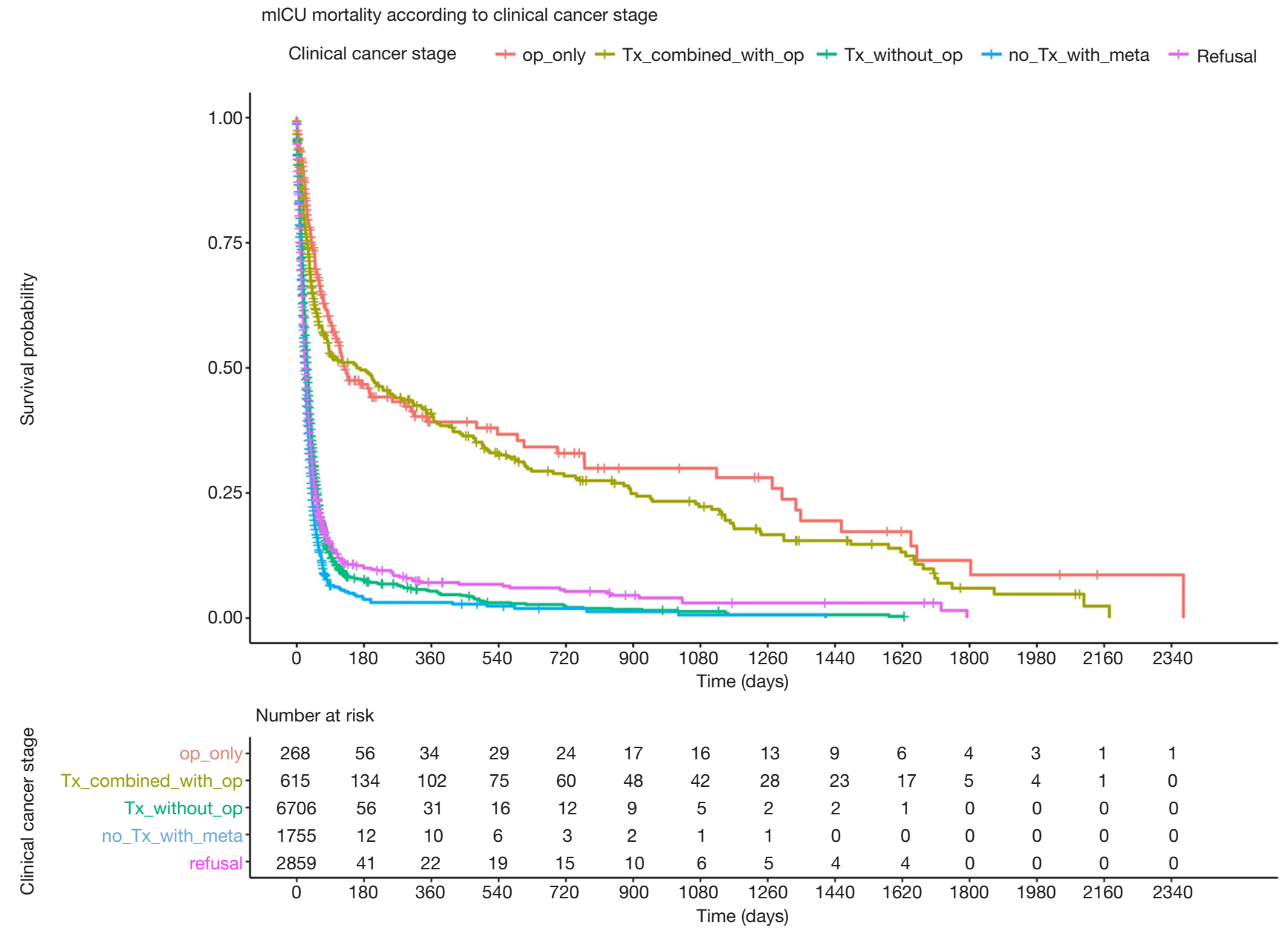

Figure 2 Intensive care unit admission for nonsurgical reasons according to clinical stage. Op_only, lung cancer resection only; Tx_ combined_with_op, combined systemic therapy with lung cancer resection; Tx_without_op, systemic therapy without lung cancer resection; no_Tx_with_meta, no treatment; refusal, refusal of any treatment.

for patients with lung cancer in admitting to ICU using the US Medicare registry (17). Patients who received MV went could discharge with less than $20 \%$ and survived with only $15 \%$ after discharge, while the overall survival had not improved from 1992 to 2007.

A multinational study was reported for the outcomes of ICU admission in patients with lung cancer (12). The study suggested that patients not fit for chemotherapy or patients with poor performance might be better to receive palliative care than ICU care in critically ill states. Our study has several strengths compared to above large scaled or population based studies. First, we evaluated a national claim data for Korean general population which all people are covered and followed up long terms of 5 years. Second, we separated from post-operative ICU admission known to result good prognosis. On these strengths, it can lead for physicians to decide more carefully and discuss in detail for whether to proceed ICU care including intubation and mechanical ventilation in patients with lung cancer.

There are several limitations to consider our results. First, potential selection bias could interfere results of our study. This study was not planed with prospective and randomized control design, and did not use propensity matching groups. However, this study could show comprehensive insights for ICU outcomes in critically ill patients with lung cancer, using a national population-based cohort, as like other national studies with important impact $(12,17)$. Second, we used administrative reimbursement claim data for this study. The data did not include any information on the pathologic and radiologic findings of 
lung cancer. We could not differentiate between small cell carcinoma and non-small cell carcinoma. In our study, TNM staging system could not be applied due to the innate limitations of claim data. The TNM stage at the time of diagnosis is important for planning future therapy and predicting the prognosis at the time of diagnosis. However, the cancer treatments that the patient actually received before being admitted to ICU are more important in the prognosis of intensive care. Third, we could not obtain the code status and performance status of lung cancer patients. Therefore, our results may have included futile critical care, with no clear distinction between treatment groups. However, these results have implications for understanding and assessing the actual critical care as not all hospitals are well-skilled and systematic in the treatment of critically ill patients with lung cancer. Despite these limitations, we have identified prognostic factors for ICU outcomes in critically ill patients with lung cancer in large sample size, population-based dataset

\section{Conclusions}

It is vital for physicians involved in critical care to understand factors influencing clinical outcomes of medical ICU in order to avoid futile treatment in patients with lung cancer and to use limited medical resources more effectively (1-3). Our study confirms that clinical cancer staging is the most important factor in predicting outcomes in critically ill patients with lung cancer, and that the need for a mechanical ventilator contributes to prognostic factors especially in early lung cancer patients. Further studies of prognostic factors for each stage of lung cancer are needed to establish appropriate critical care directions.

\section{Acknowledgments}

Funding: This study was supported by 2019 Research Grant from Kangwon National University and a 2018 Research Grant from Kangwon National University Hospital.

\section{Footnote}

Reporting Checklist: The authors have completed the STROBE reporting checklist. Available at http://dx.doi. org/10.21037/atm-21-298

Data Sharing Statement: Available at http://dx.doi. org/10.21037/atm-21-298
Peer Review File: Available at http://dx.doi.org/10.21037/ atm-21-298

Conflicts of Interest: All authors have completed the ICMJE uniform disclosure form (available at http://dx.doi. org/10.21037/atm-21-298). The authors have no conflicts of interest to declare.

Ethical Statement: The authors are accountable for all aspects of the work in ensuring that questions related to the accuracy or integrity of any part of the work are appropriately investigated and resolved. The study was conducted in accordance with the Declaration of Helsinki (as revised in 2013). The study was approved Kangwon National University Hospital Institutional Review Board (B2018-02-002) and individual consent for this retrospective analysis was waived.

Open Access Statement: This is an Open Access article distributed in accordance with the Creative Commons Attribution-NonCommercial-NoDerivs 4.0 International License (CC BY-NC-ND 4.0), which permits the noncommercial replication and distribution of the article with the strict proviso that no changes or edits are made and the original work is properly cited (including links to both the formal publication through the relevant DOI and the license). See: https://creativecommons.org/licenses/by-nc-nd/4.0/.

\section{References}

1. Ferlay J, Colombet M, Soerjomataram I, et al. Estimating the global cancer incidence and mortality in 2018:

GLOBOCAN sources and methods. Int J Cancer 2019;144:1941-53.

2. Soares M, Caruso P, Silva E, et al. Characteristics and outcomes of patients with cancer requiring admission to intensive care units: a prospective multicenter study. Crit Care Med 2010;38:9-15.

3. Kress JP, Christenson J, Pohlman AS, et al. Outcomes of critically ill cancer patients in a university hospital setting. Am J Respir Crit Care Med 1999;160:1957-61.

4. Roques S, Parrot A, Lavole A, et al. Six-month prognosis of patients with lung cancer admitted to the intensive care unit. Intensive Care Med 2009;35:2044-50.

5. Oeyen SG, Benoit DD, Annemans L, et al. Long-term outcomes and quality of life in critically ill patients with hematological or solid malignancies: a single center study. Intensive Care Med 2013;39:889-98. 
6. Kochanek M, Schalk E, von Bergwelt-Baildon M, et al. Management of sepsis in neutropenic cancer patients: 2018 guidelines from the Infectious Diseases Working Party (AGIHO) and Intensive Care Working Party (iCHOP) of the German Society of Hematology and Medical Oncology (DGHO). Ann Hematol 2019;98:1051-69.

7. Azoulay E, Schellongowski P, Darmon M, et al. The Intensive Care Medicine research agenda on critically ill oncology and hematology patients. Intensive Care Med 2017;43:1366-82.

8. Soubani AO, Ruckdeschel JC. The outcome of medical intensive care for lung cancer patients: the case for optimism. J Thorac Oncol 2011;6:633-8.

9. Reck M, Rabe KF. Precision Diagnosis and Treatment for Advanced Non-Small-Cell Lung Cancer. N Engl J Med 2017;377:849-61.

10. Gettinger S, Horn L, Jackman D, et al. Five-Year FollowUp of Nivolumab in Previously Treated Advanced NonSmall-Cell Lung Cancer: Results From the CA209-003 Study. J Clin Oncol 2018;36:1675-84.

11. Azoulay E, Soares M, Darmon M, et al. Intensive care of the cancer patient: recent achievements and remaining challenges. Ann Intensive Care 2011;1:5.

12. Soares M, Toffart AC, Timsit JF, et al. Intensive care in patients with lung cancer: a multinational study. Ann Oncol 2014;25:1829-35.

Cite this article as: Park J, Kim WJ, Hong JY, Hong Y. Clinical outcomes in patients with lung cancer admitted to intensive care units. Ann Transl Med 2021;9(10):836. doi: 10.21037/atm-21-298
13. Park J, Jeon K, Chung CR, et al. A nationwide analysis of intensive care unit admissions, 2009-2014 - The Korean ICU National Data (KIND) study. J Crit Care 2018;44:24-30.

14. Hickey GL, Dunning J, Seifert B, et al. Statistical and data reporting guidelines for the European Journal of Cardio-Thoracic Surgery and the Interactive CardioVascular and Thoracic Surgery. Eur J Cardiothorac Surg 2015;48:180-93.

15. Reichner CA, Thompson JA, O'Brien S, et al. Outcome and code status of lung cancer patients admitted to the medical ICU. Chest 2006;130:719-23.

16. Adam AK, Soubani AO. Outcome and prognostic factors of lung cancer patients admitted to the medical intensive care unit. Eur Respir J 2008;31:47-53.

17. Slatore CG, Cecere LM, Letourneau JL, et al. Intensive care unit outcomes among patients with lung cancer in the surveillance, epidemiology, and end results-medicare registry. J Clin Oncol 2012;30:1686-91.

18. Darmon M, Bourmaud A, Georges Q, et al. Changes in critically ill cancer patients' short-term outcome over the last decades: results of systematic review with meta-analysis on individual data. Intensive Care Med 2019;45:977-87.

19. Puxty K, McLoone P, Quasim T, et al. Risk of Critical Illness Among Patients With Solid Cancers: A PopulationBased Observational Study. JAMA Oncol 2015;1:1078-85. 